Bryn Mawr College

Scholarship, Research, and Creative Work at Bryn Mawr

College

1985

Low-Dimensional Chaotic Attractors for an Unstable, Inhomogeneously Broadened, SingleMode Laser

Alfonso M. Albano

Bryn Mawr College, aalbano@brynmawr.edu

J.Abounadi

T. H. Chyba

C. E. Searle

S. Yong

See next page for additional authors

Let us know how access to this document benefits you.

Follow this and additional works at: http://repository.brynmawr.edu/physics_pubs

Part of the Physics Commons

Custom Citation

A.M. Albano et al, J. Opt. Soc. Am. B. 2, 47 (1985).

This paper is posted at Scholarship, Research, and Creative Work at Bryn Mawr College. http://repository.brynmawr.edu/physics_pubs/23

For more information, please contact repository@brynmawr.edu. 
Authors

Alfonso M. Albano, J. Abounadi, T. H. Chyba, C. E. Searle, S. Yong, R. S. Gioggia, and N. B. Abraham 


\title{
Low-dimensional chaotic attractors for an unstable, inhomogeneously broadened, single-mode laser
}

\author{
A. M. Albano, J. Abounadi, T. H. Chyba, ${ }^{*}$ C. E. Searle, and S. Yong
}

Department of Physics, Bryn Mawr College, Bryn Mawr, Pennsylvania 19010

R. S. Gioggia

Department of Physics, Widener University, Chester, Pennsylvania 19013

\author{
N. B. Abraham ${ }^{\dagger}$
}

Istituto Nazionale di Ottica, Largo Enrico Fermi 6, 50125 Firenze, Italy

Received August 15, 1984; accepted September 24, 1984

\begin{abstract}
Quantitative characterization of the intensity pulsations from an inhomogeneously broadened laser confirm that observed irregular pulsing has its origins in deterministic chaos corresponding to motion on a strange attractor of low fractal dimensionality. The pointwise information dimension and the Grassberger-Procaccia $K_{2}$ (estimators from below of the fractal dimensionality of the attractor and the Kolmogorov entropy, respectively) have been determined for digitized time series from parameter regions identified qualitatively by power spectra as representing periodic, period-doubled, quasi-periodic, and chaotic behavior. Some amount of chaos seems present for almost all operating conditions.
\end{abstract}

\section{INTRODUCTION}

Advances in theoretical understanding of the behavior of dissipative nonlinear dynamical systems far from thermodynamic equilibrium have provided a basis for describing certain kinds of irregular asymptotic evolution as deterministic chaos. ${ }^{1}$ Key features of such chaos are irregular time evolution and broadband power spectra, which are nevertheless associated with deterministic motion on an attracting subspace of the underlying physical variables. These attractors are called strange attractors, in part because evolution is sensitively dependent on initial conditions and the attracting subspace has fractal dimensionality.

Qualitatively, one can identify the likely presence of an underlying strange attractor when irregular time dependence and broadband power spectra appear for a system that was evolving in an obviously deterministic fashion (such as with output that was constant or strictly periodic) for nearby parameter values. Such identifications of chaos are reinforced if the system is observed to make one of the relatively universal progressions of increasing complexity as a parameter is slowly changed [e.g., period doubling (the sequential appearance of subharmonics to the original pulsing frequency) or quasi-periodicity (the successive appearance of two incommensurate frequencies)] before the appearance of irregular pulsing and broadband spectra.

Following these prescriptions, we recently studied unstable, single-mode, inhomogeneously broadened lasers for various operating conditions. ${ }^{2}$ Measurements of the temporal and spectral output characteristics have led to the identification of regimes of chaotic behavior. Chaotic behavior in lasers is not unexpected, as analogies have been established between simple models for single-mode and multimode homogeneously broadened lasers ${ }^{3,4}$ and the Lorenz equations, ${ }^{5}$ which were designed for the study of convective instabilities in hydrodynamics and which were shown to contain deterministic chaos among their possible solutions. However, the temporal evolution of inhomogeneously broadened lasers, which have experimentally accessible regions of unstable operation, ${ }^{6}$ has been modeled successfully only by extremely large numbers (hundreds) of coupled equations, ${ }^{7}$ and the origin or nature of chaotic solutions is not yet clearly established.

In our previous experimental identification of chaotic behavior using qualitative measures, we were not able to distinguish unambiguously between low-dimensional deterministic chaos and broadband stochastic noise originating from the contributions of many uncorrelated sources. Such noisy behavior may arise from direct multisource contributions or because the deterministic states of the system are relatively unstable (or only weakly stable) and act as sensitive amplifiers of noisy perturbations.

Quantitative methods of analyzing digitized time series to distinguish stochastic noise from deterministic chaos were

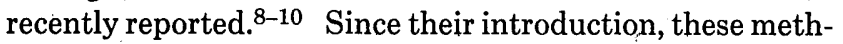
ods have been refined, greatly reducing the data requirements for obtaining reliable estimates of such characteristic properties as Lyapunov exponents, metric entropies, and attractor dimensionalities. ${ }^{11}$ The reduced data requirements make it possible to apply these techniques to the kinds of digitized data that can be obtained from our low-power lasers with their high pulsing frequencies. An added benefit of these techniques is that, when noise acts as a simple additive diffusion, either originating in the laser system (e.g., mechanical disturbances of the laser or spontaneous emission ${ }^{12,13}$ ) or arising 
from the detection electronics, it can be separated from dynamical behavior if the noise blurring is small compared with the characteristic dynamical features. ${ }^{9}$ Another advantage of these techniques is that recording of a single digitized variable of the system permits the reconstruction of the topological character of the attractor in the total variable space. This is accomplished by embedding the time series in an $N$-dimensional vector space in which each vector has components that are data values taken with equal time-delay separations. ${ }^{14-16}$ By the Whitney embedding theorem, ${ }^{14}$ the attractor constructed in a sufficiently large embedding space has the same topological structure as the true attractor in the variable space (that is, it will have the same Lyapunov exponents, metric entropies, and dimensionalities).

A good introduction to these measurement and analysis techniques can be found in descriptions of their recently successful application in the study of low-frequency instabilities in hydrodynamic systems. ${ }^{17}$ Here, we demonstrate the robustness of the methods by moving to systems that differ by nearly $10^{8}$ in the fundamental pulsing frequency.

\section{EXPERIMENTAL SYSTEM}

The laser system studied was that previously described 2,18 that uses the high-gain 3.51- $\mu \mathrm{m}$ laser transition in xenon. The population inversion is created by a dc discharge in the laser tube controlled by a well-regulated high-voltage power supply and a series ballast resistor, which effectively suppress fluctuations in the excitation process.

The intensity output was digitized by a Tektronix fast transient digitizer, which provided 10-bit resolution and sequences of up to 512 data points. The data sets were stored by an Apple microcomputer and were transferred to a Burroughs 6900 computer for analysis.

For fixed pressure and discharge current, data sets were taken for different cavity detunings, which gave qualitatively different power spectra. The pressure mixture used was 180 mTorr of xenon (99\% enriched ${ }^{136} \mathrm{Xe}$ ) and $600 \mathrm{~m}$ Torr of helium at an excitation current of $4 \mathrm{~mA}$, a particular parameter set from the complete survey of the laser operating conditions reported elsewhere. ${ }^{18}$

Control data sets were taken by supplying a sine-wave input to the digitizer (thus retaining any systematic errors that were due to the digitizer) and by computer generation of periodic, quasi-periodic, and random data sets with suitable resolution and data-set length.

\section{RESULTS}

Figures 1 and 2 show two typical data sets, with a portion of the digitized time series shown in Figs. 1(a) and 1(b) and the associated intensity power spectrum from a scanning, timeaveraging rf-spectrum analyzer shown in Figs. 1(b) and 2(b). The sampling interval $\tau$ for Figs. 1(a) and 2(a) was set to 1 nsec. In Figs. 1(c) and 2(c) we give, for visualization, a sample two-dimensional embedding of the data $[x(t)$ versus $x(t+p \tau)$ for $p=10]$.

In Figs. 1(d) and 2(d) we plot curves following the prescription of Procaccia and Grassberger, ${ }^{9}$ namely, for each embedding dimension $q$, a vector is defined as $x(t), x(t+p \tau)$, $x(t+2 p \tau), \ldots, x[t+(q-1) p \tau]$, where $p$ is an integer and $\tau$ is the sampling interval. Then we determine the total number of distances between these vectors that are less than $\epsilon$, and we plot this number $N(\epsilon)$ versus $\epsilon$ in a $\log -\log$ plot for embedding dimensions 1-10, as shown in Figs. 1(d) and 2(d). Although for simplicity this is not shown, we continue the process of calculations and plots up to embedding dimension 20.

To interpret these data, we refer to Fig. 3, where we have the corresponding results for the digitized sine wave. The $\log -\log$ plot of $N(\epsilon)$ versus $\epsilon$ shows three distinct regions for each dimension. For $\epsilon$ large enough to exceed the largest interpoint distance, $N(\epsilon)$ saturates at a constant $N_{p q}(\infty)$, the total number of interpoint distances given by $N_{p q}(\infty)=[N$ $-(q-1) p](N-q p) / 2$, where $N$ is the total number of data points and $q$ and $p$ are as defined above. For small $\epsilon$, the distances are blurred by noise sources, so the dependence of $\mathrm{N}(\epsilon)$ on $\epsilon$ increases with increasing embedding dimension. For intermediate $\epsilon$ values, we expect to find that $N(\epsilon)$ scales as $\epsilon^{\nu}$, when the embedding dimension is large enough to reflect fully the topological character of the strange attractor, where $\nu$ is defined by Grassberger and Procaccia ${ }^{9}$ as the pointwise information dimension and is an estimator from below for the fractal dimensionality of the attractor. For a simple periodic signal we expect $\nu=1$, while quasi-periodic signals from a mixture of two incommensurate frequencies should give $\nu=$ 2 and chaotic signals should give $\nu>2$.

Figures 1(e), 2(e), and (3e) show the slope $\nu$ of the log-log plots versus $\log N(\epsilon)$ for dimensions 1-10, and Figs. 1(f), 2(f), and $3(\mathrm{f})$ show these results for dimensions 11-20. These figures were drawn to determine those regions in the $\log _{e} N(\epsilon)$ versus $\log _{e}(\epsilon)$ plots that are relatively free of the effects of both noise [low $\epsilon$ or low $N(\epsilon)$ ] and saturation [high $\epsilon$ or high $N(\epsilon)$ ]. Since there are crucial restrictions imposed by our relatively small number of data points, we chose to seek regions defined by $\log _{e} N$ rather than $\log _{e}(\epsilon)$, as was done, for instance, by Brandstatter et $a l .{ }^{17}$ In Figures 3(d) and 3(f), the intermediate regions of $\epsilon$ [for $\log _{e} N(\epsilon)$ between 8.5 and 9.5], we see that the curves approach the same slope with a value of $1.0 \pm 0.1$. The values converge rapidly with increasing embedding dimension, although we see that $10 \%$ accuracy is achieved only for dimensions 11 and larger. We have indications from various control data sets that a small overestimate may occur because of the limited number of data points and the finite resolution of the digitizer, yet these results show powerful quantitative accuracy for the method when applied to data sets with our experimental limitations.

Procaccia and Grassberger also define an entropy $K_{2}$ (Ref. 9 ), which can readily be extracted from these plots and which provides an estimate from below for the Kolmogorov entropy $K$. The useful relationships are that $K_{2}$ and $K$ should be zero for periodic (ordered) signals, finite (and nonzero) for chaotic attractors, and infinite for stochastic random noise. In the limit of a sufficiently large embedding dimension to recover the attractor topology, the spacing between the parallel line parts of the $\log -\log$ plots of $N(\epsilon)$ versus $\epsilon$ should be given by $K_{2} p \tau$, where $p \tau$ is the delay time used in the embedding procedure. [This is strictly true in the limit $\epsilon \rightarrow 0$ and if instead of $N(\epsilon)$ one plots a true probability $N(\epsilon) / N(\infty)$ in each case.] As our plots are made for $N(\epsilon)$, and $N(\epsilon)$ changes with increasing embedding dimension, we obtain values of $K_{2} p \tau$ overestimated by $0.004 p$. Here we calculate $K_{2}(\epsilon) p \tau$, the separations at finite $\epsilon$.

Figure 4(a) shows the values of the vertical separation of the curves of Figs. 1(d), 2(d), 3(d) and their extensions to higher dimensions versus the embedding dimension for the two data 

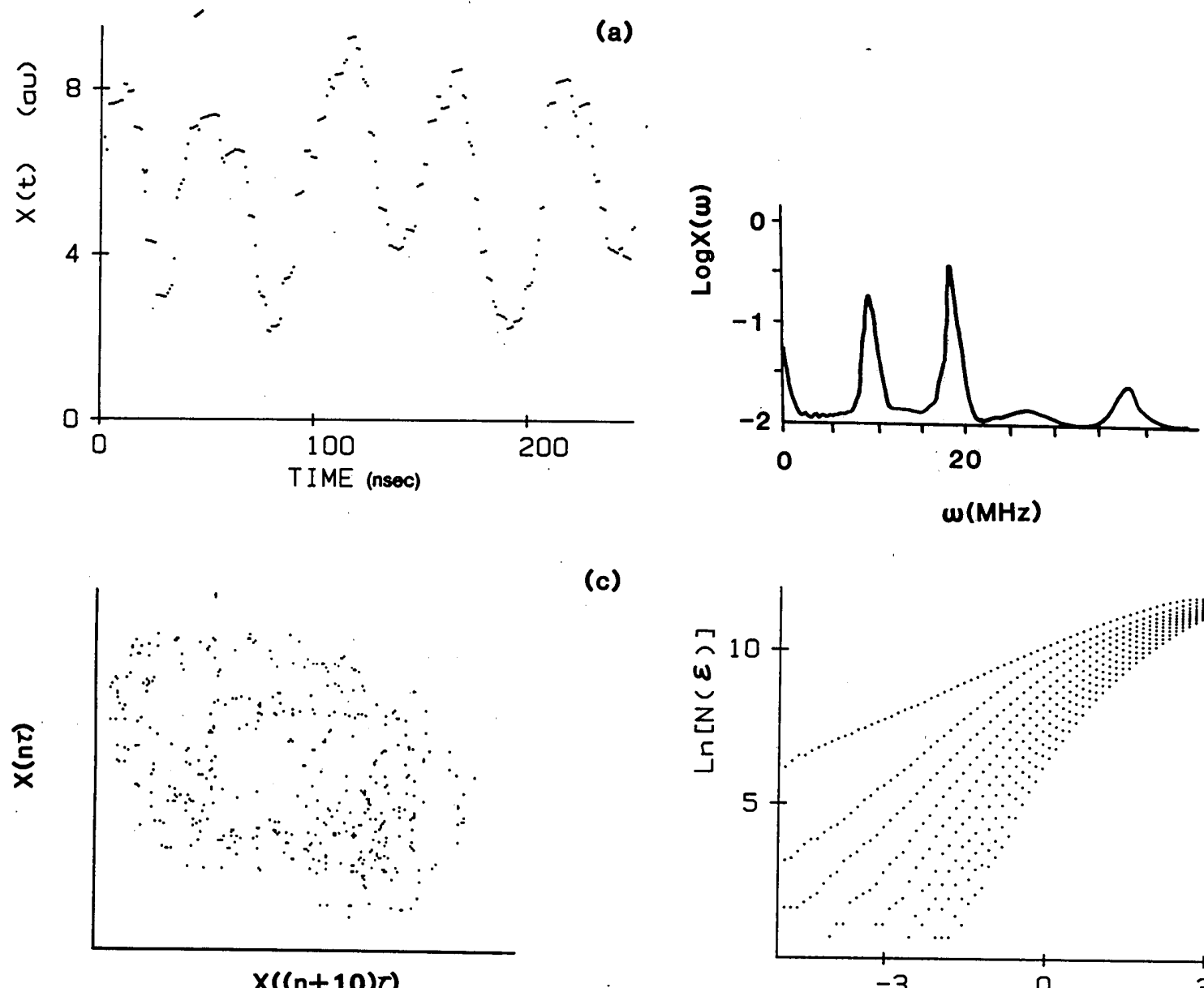

(c)

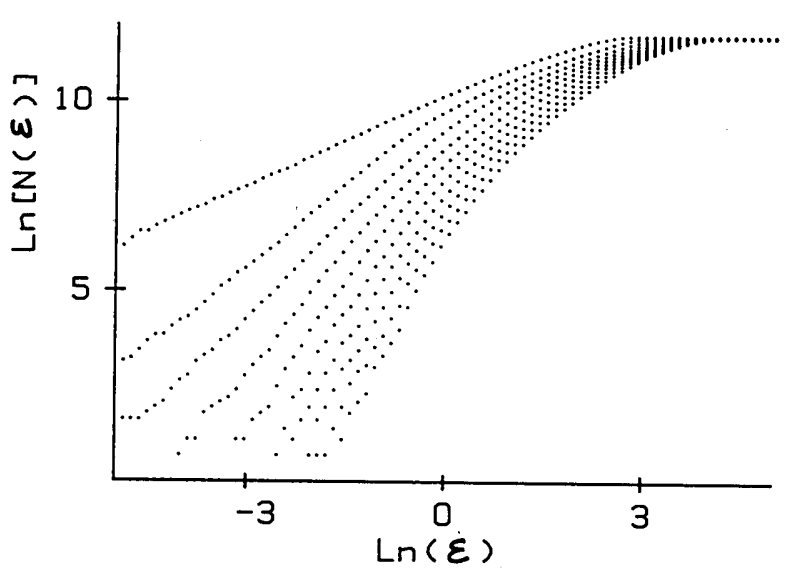

(d)

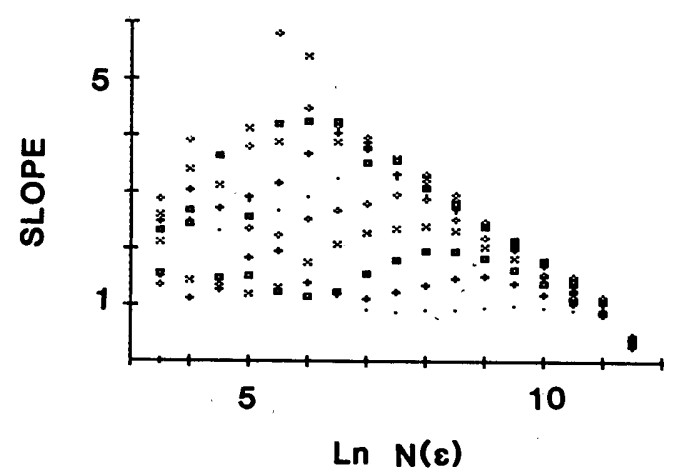

(e)

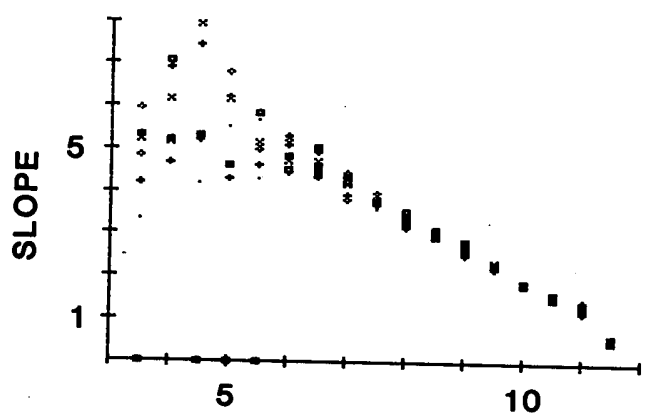

(f)

Fig. 1. Analysis of data set A9 $\left(V_{\mathrm{PZT}}=1.58 \times 0.3 \mathrm{kV}\right)$. (a) Part of the digitized data set plotted as a function of time. (b) Power spectrum

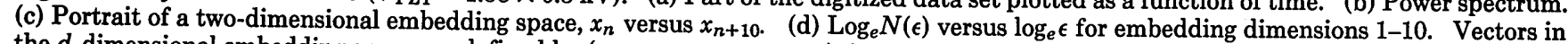
the $d$-dimensional embedding space are defined by $\left(x_{k}, x_{k+1}, \ldots, x_{k+d-1}\right)$, i.e., $p=1$. (e) Slope of $\log _{e} N(\epsilon)$ versus $\log _{e} \epsilon$ curves as functions of $\log _{e} N(\epsilon)$ for embedding dimensions 1-10. (f) Same as (e) for embedding dimensions 11-20. Note change in vertical scale.

sets shown in Figs. 1 and 2 as well as for the digitized sinusoidal signal shown in Fig. 3, all taken at $\log _{e}(\epsilon)=1.9$.

Also included are two sets of results for 500 random numbers generated by an Apple Ile. The asterisks marked 1.9 were calculated at $\log _{e}(\epsilon)=1.9$; the unlabeled asterisks, at $\log _{e}(\epsilon)=3.3$.

The values obtained for the random signal at $\log _{e} \epsilon=3.3$ are flawed for low embedding dimensions by the fact that, here, some of the $\log _{e}(N)$ versus $\log _{e}(\epsilon)$ curves have already begun to saturate. The values obtained at $\log _{e}(\epsilon)=1.9$, on the other hand, are unreliable at higher embedding dimensions since here one deals with a relatively small number of points. Thus we show results only for embedding dimensions 1-5. In fact, for embedding dimensions greater than 10 there are no pairs of points with separations less than $\epsilon=e^{1.9}$. Nevertheless, one sees that the values of $K_{2}(\epsilon)$ for the random signal tend to much higher levels than those for the other data, and there is no indication at all that they tend toward a limit.

The plot for the sinusoidal signal shows evidence of progression toward zero reaching a value of 0.047 , which appears to us to be the limit possible with the effective 10-bit digitizing accuracy in our sampling of the signal. The values of $K_{2} p \tau$ 

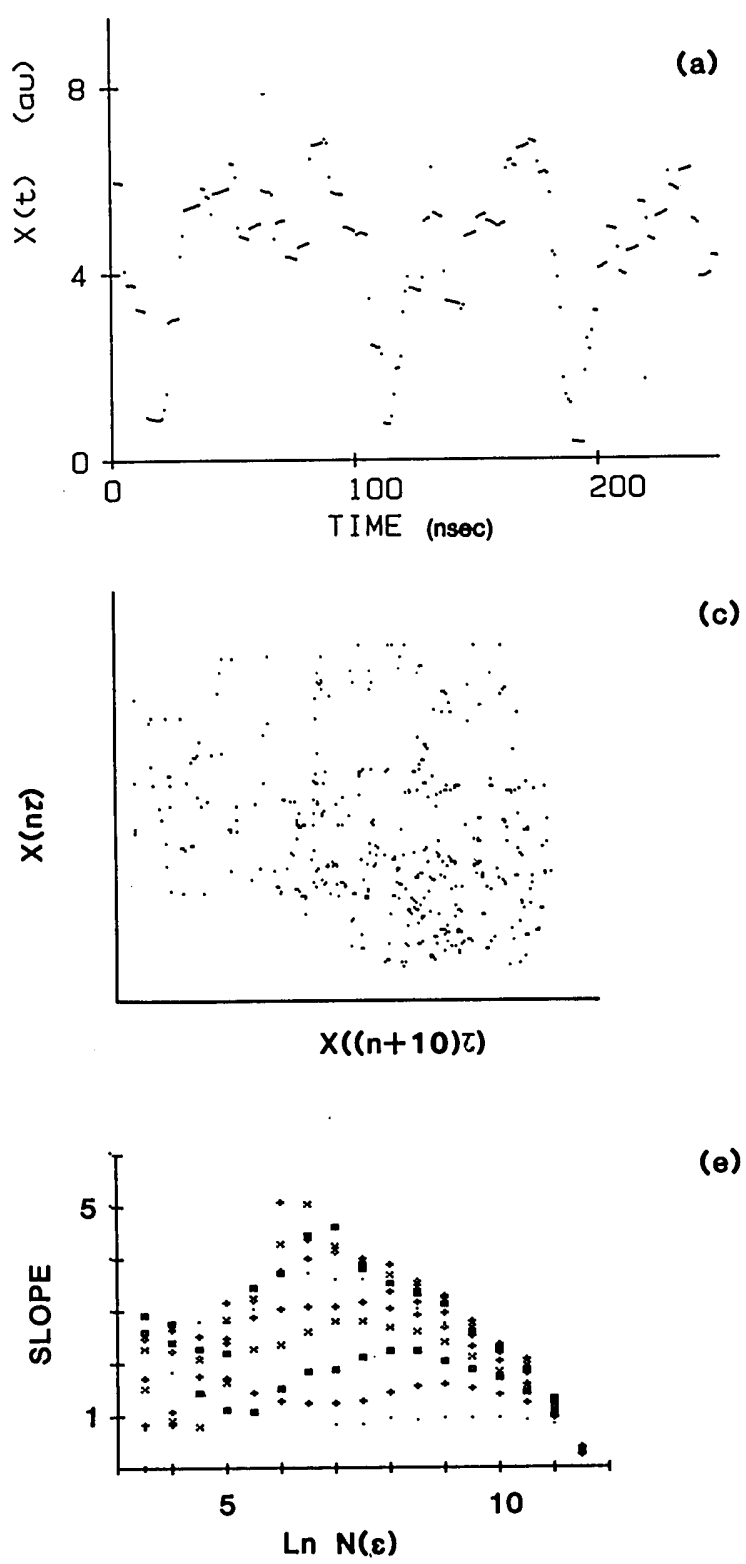

(c)

(e) (b)
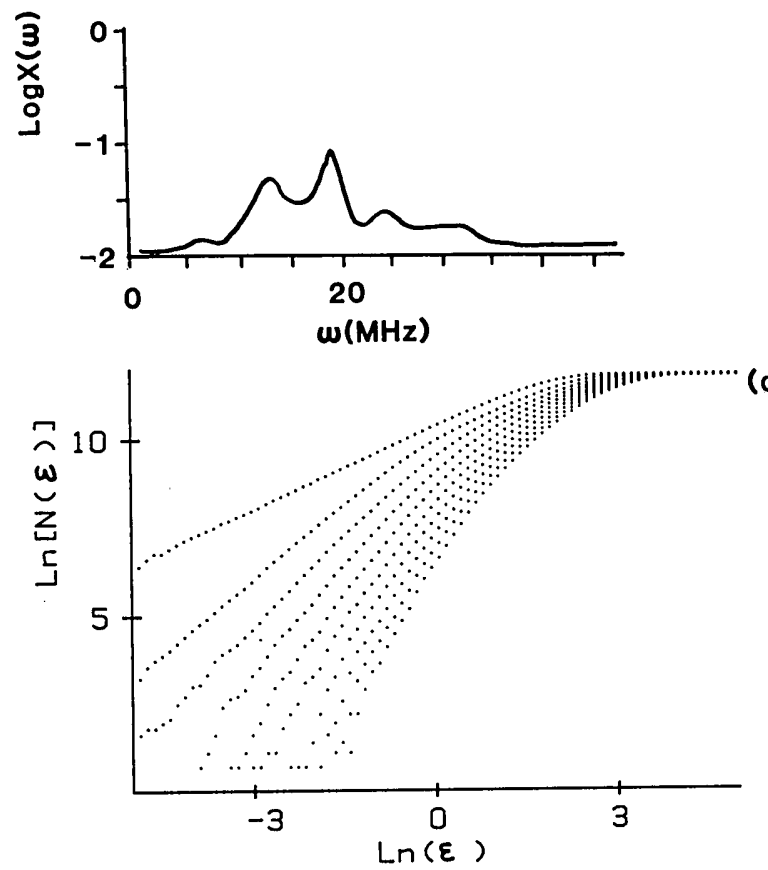

(d)

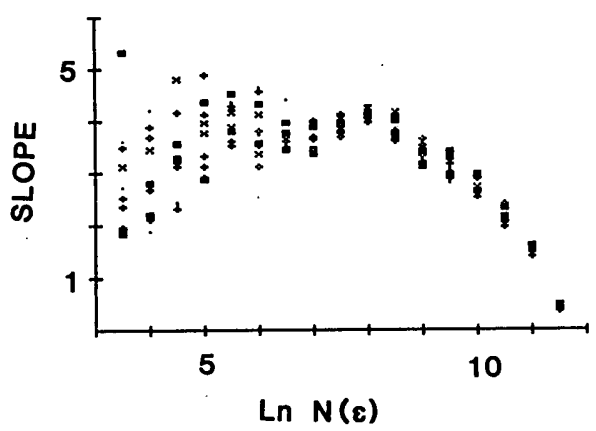

Fig. 2. Analysis of data set $\mathrm{I} 4\left(V_{\mathrm{PZT}}=1.82 \times 0.3 \mathrm{kV}\right)$. Figures correspond to those in Fig. 1 except that for $(\mathrm{e})$ and (f) vectors in the $d$-dimensional embedding space are defined by $\left(x_{k}, x_{k+2}, \ldots, X_{k+2 s-2}\right)$, i.e., $p=2$.

for the two data sets are distinctly above the value for the sinusoidal signal with values (corrected for the offset) of 0.175 for the results of Fig. 1 and 0.190 for the results of Fig. 2 when taken at dimension 19 , and these seem to be converging to values above the value for the sine-wave data. These nonzero values suggest that the data sets are taken from a chaotic operating condition, a result that is consistent with the corresponding values of the dimension that can be extracted from Fig. 4(b). Here, we see that the slope converges with increasing dimension, just as the curve separation did. The asymptotic values are greater than 2 , again suggesting chaotic behavior.

Returning to the data sets in Figs. 1 and 2, we again see that for sufficiently large $N(\epsilon)$ the values of the slopes coalesce, becoming independent of the embedding dimension. The plateau regions of these coalesced values are smaller than in the sine-wave case because of the poorer signal-to-noise ratio of the low-power laser output compared with the noise con- tributions of the detector, preamplifier, and digitizer. Apparently noise and signal are resolved for high dimensions, but the continuous decline of the slope with increasing $N(\epsilon)$, where the curves have converged (rather than a strict plateau), signals that we may underestimate the true attractor dimensionality by taking the value of convergence. We cannot achieve a better noise rejection with our limited number of points. Variations of the delay (controlled by $p$ ) in defining the vectors were found to make only minor qualitative and quantitative changes in these results.

Summarizing many data sets for each parameter value and a wide variety of different cavity detunings, we plot in Fig. 5 the laser-power output, the frequencies of the principal peaks in the power spectrum, and the values of $\nu$ and $K_{2}(\epsilon) \tau$ for each setting. The error bars indicate the range of results for different data sets.

From the frequencies we can identify regions that might be classified qualitatively as periodic, period-doubled ( $P 2$ and 
P4), chaotic, and quasi-periodic (two incommensurate frequencies). The dimensionalities and entropies do not provide equally simple qualitative distinctions, but there are strong correlations.

The dimensionalities are consistently in the range $2-4$, with noticeable increases in the chaotic region. The fact that operating regions that result in seemingly periodic signals (only one principal power-spectrum peak and its harmonics) have attractor dimensionalities of order 2 instead of 1 is not fully explained. Tests of computer-generated data do not show such a large discrepancy, even when one takes into account the noise levels and the brevity of the data sets. However, it is not yet theoretically established that there are regions of

(a)
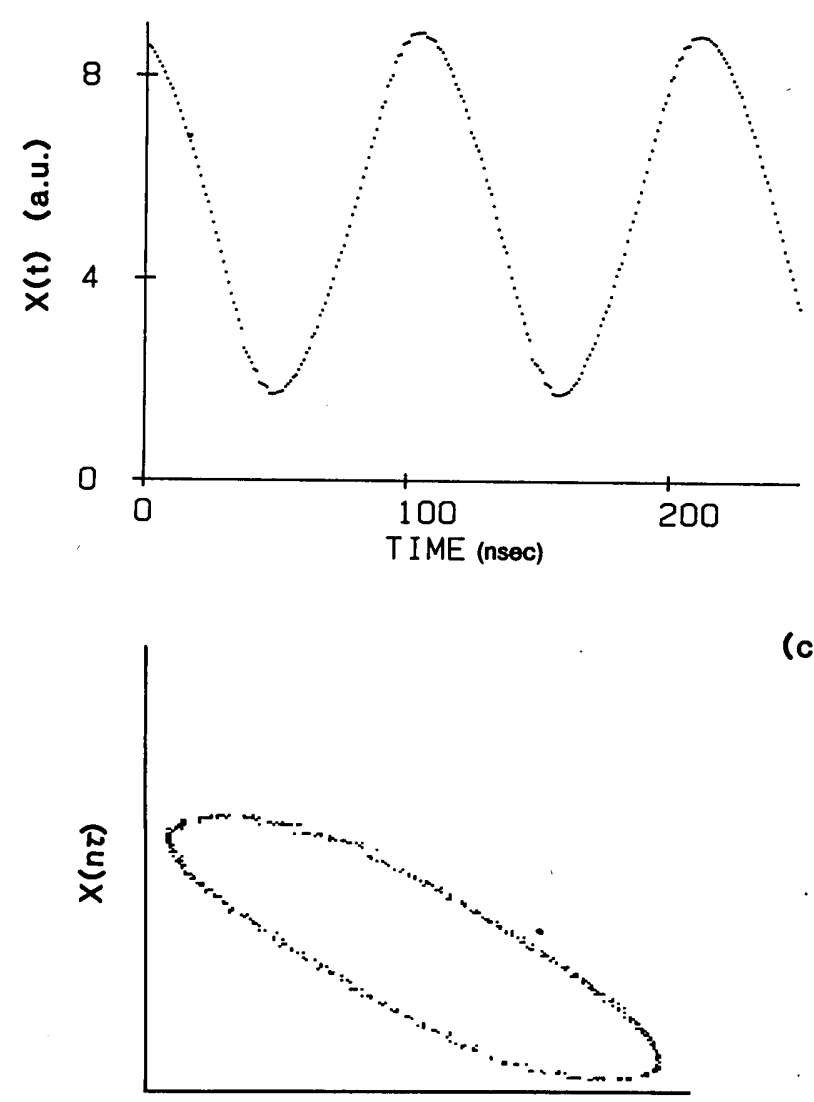

$x((n+10) \tau)$

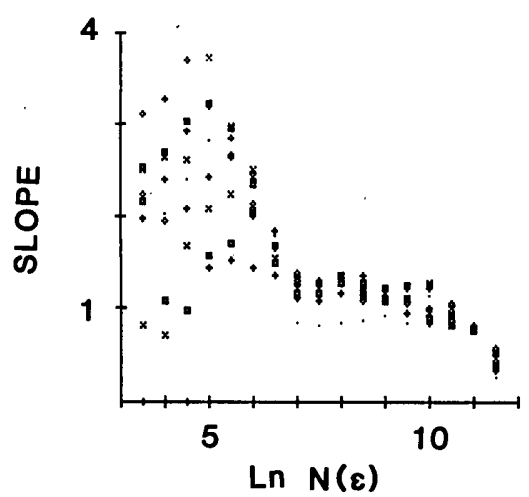

(e)

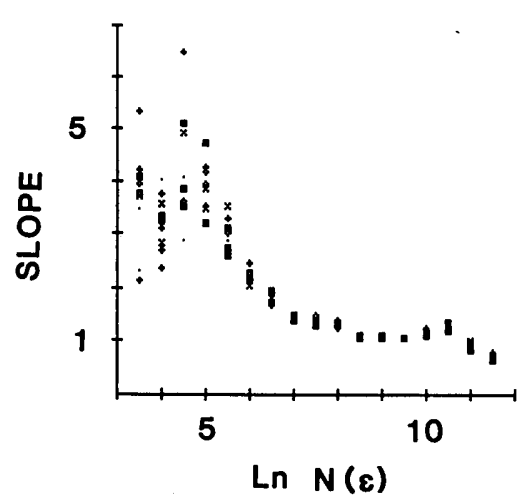

(f) entirely stable periodic pulsations for this type of laser, so we may simply be making qualitative distinctions from the power spectra between regions of more-or-less periodic chaos.

Close examination of the plot of peak frequencies in Fig. 5 reveals many subtleties that might be overlooked in qualitatively discussing a single power spectrum. In the center and at the right are two regions of broadband spectra with apparent period-doubling sequences leading to them ( $P 1-P 2-$ $P 4-C$ appears from $V_{\mathrm{PZT}}=1.95$ to 1.85 , and $P 1-P 2-C 2-P 2$ appears for $V_{\mathrm{PZT}}=1.75$ to 1.8 . The dimensionality calculation shows a sharp jump in the dimension when these sequences converge to the chaotic regions. If our identifications are quantitatively accurate, we would expect dimension 1 for (c)

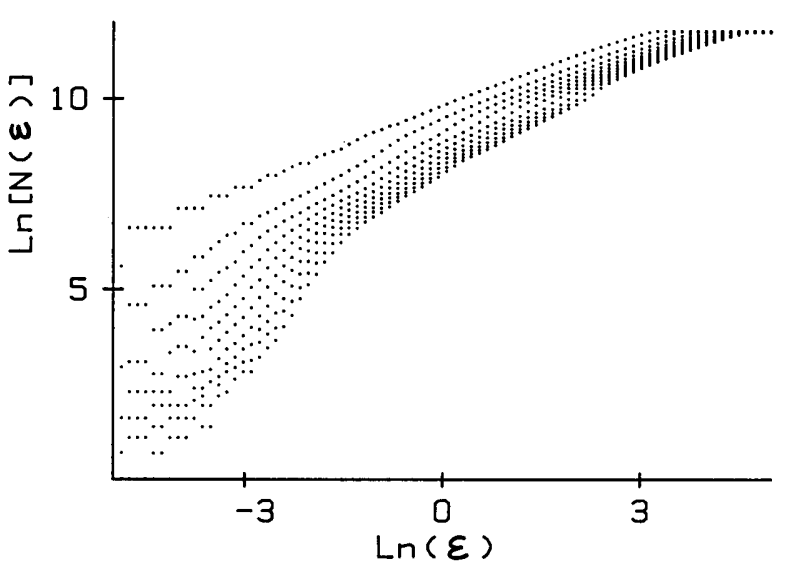

(d)

Fig. 3. Analysis of digitized sinusoidal signal of 2-MHz frequency. Figures correspond to those in Fig. 1. 


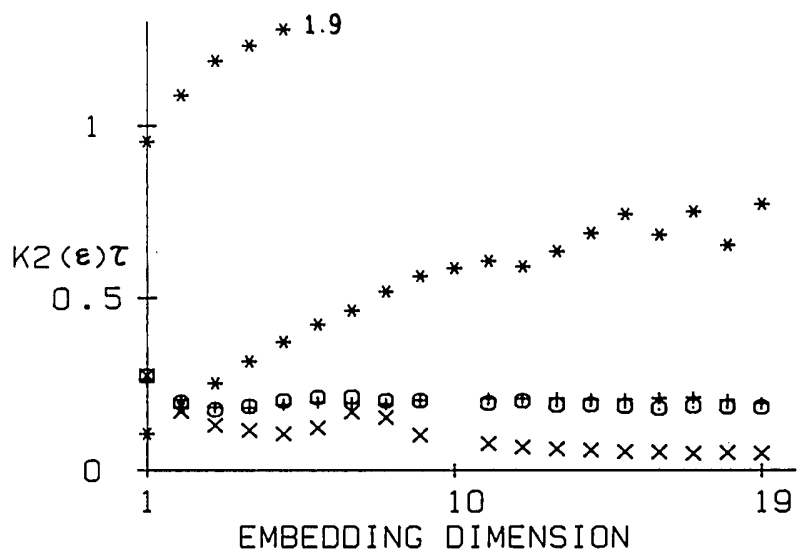

(a)

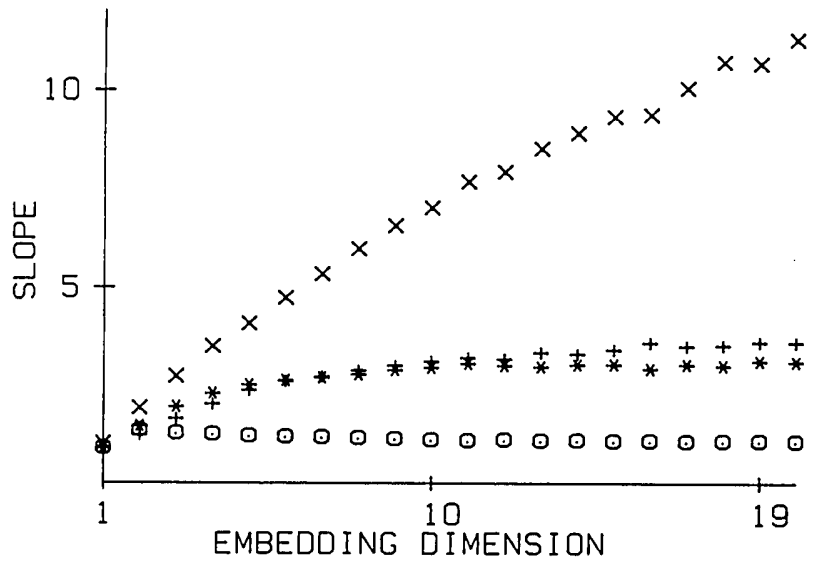

(b)

Fig. 4. (a) Vertical separation $K_{2}(\epsilon) \tau$ of the curves in Figs. 1(d), 2(d), and $3(\mathrm{~d})$ (taken at $\log _{e} \epsilon=1.9$ ) versus embedding dimension for data sets A9, I4, and sine and for a set of random numbers. The asterisks show the random signal, evaluated at $\log _{e} \epsilon=3.3$. Values of $K_{2} \tau$ for embedding dimension 19 are 0.18 for data set $A 9,0.19$ for data set 14 , and 0.05 for sine. These values have systematic errors of 0.004 . (b) Slopes of the plots in Figs. 1(d), 2(d), and 3(d) taken at $\log _{e} N(\epsilon)=$ 8.5.
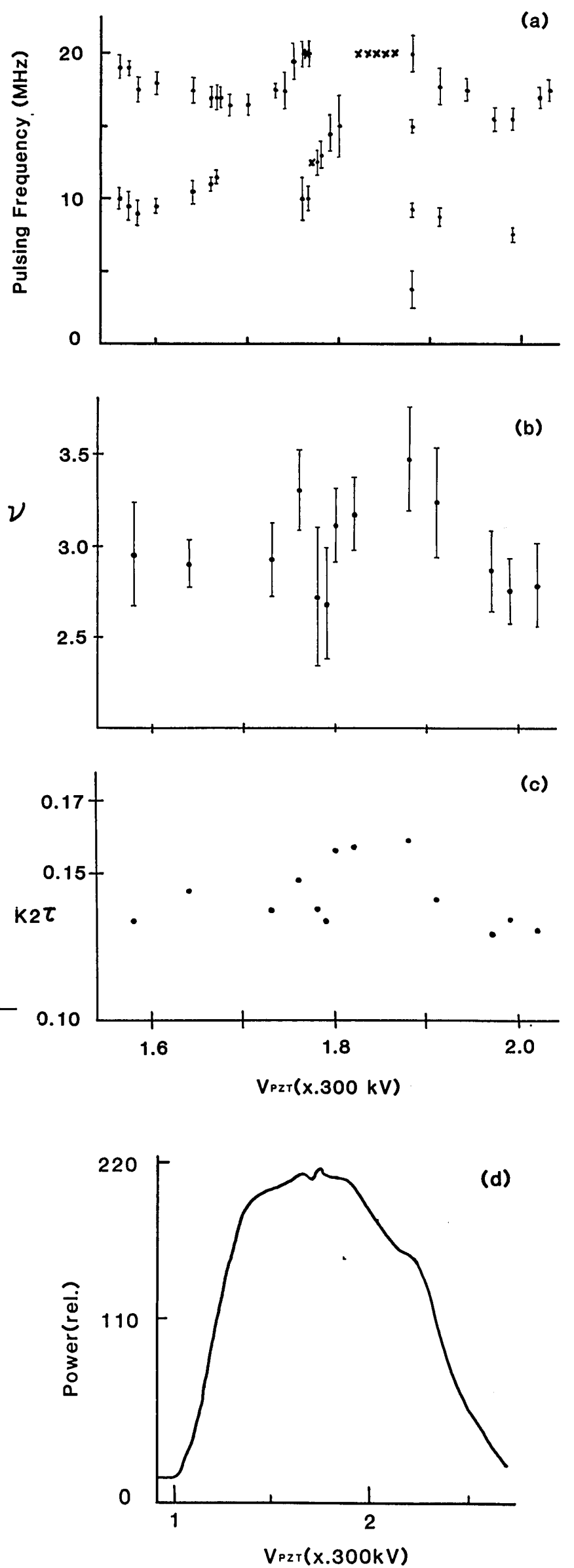

Fig. 5. (a) Principal pulsing frequencies, (b) pointwise dimension, (c) $K_{2} \tau$, and (d) average output power as functions of voltage applied to piezoelectric transducer to change the cavity length $\left(V_{\mathrm{PZT}}\right)$. In (a) the $X$ 's mark much broader spectral peaks qualitatively identified as chaotic regions. (d) Shows characteristic variations in power output identified previously in Refs. 2 and 18. 
the periodic (and the period-doubling) regions and a dimension greater than 2 for the chaos. The apparently higher values require careful examination and a further round of measurements to determine if they have their origins in measurement uncertainty. It is encouraging, however, to find the expected jump in dimensionality at the qualitatively identified transition to broader power spectra.

In the left-hand third of the tuning range in Fig. 5, there is a clear region of quasi-periodic behavior with the ratio of the frequencies varying continuously with detuning from $3: 2$ near the center to 2:1 at the far left with an apparent or near locking for the last three data values. Thus those last three spectra, which appear as a kind of period doubling if taken separately, must be identified differently as part of the region of quasiperiodicity. In the region of $V_{\mathrm{PZT}}=1.6-1.75$, where we see clearly two-frequency behavior in the power spectrum, the dimensionality is constant near a value of 3.0. Again, this is larger than the expected value (in this case 2.0), but the fact that the value is constant is consistent proof of the stability of our methods of analysis if not of their precise numerical validity.

Specific sequences to be expected in this system are not yet known because of the difficulty in modeling the complicated standing-wave effects, including overlapping hole burning in an unstable regime. Nevertheless, the dimensionality changes in ways consistent with intuitive interpretation of the power spectra.

The abrupt changes in dimensionality near the chaotic regions (near line-center tuning) are well beyond measurement uncertainties and are correlated with abrupt changes in the power spectra, samples of which are shown in Fig. 6 . We see that there are not only shifts in the qualitative nature of the spectra ( $Q P$ to $P 2$ to $P 4$ to $P 1$ ) but also abrupt shifts in the fundamental pulsing frequency that correlate with some of the abrupt changes in dimension. Probably these result from complex interaction of the counterpropagating waves through coupling to the same atoms, as can be visualized qualitatively in the overlapping of the holes burned in the gain profile. ${ }^{18}$

Research is in progress to make similar measurements of the intensity pulsations of an unstable unidirectional ring laser for which previous qualitative measurements have indicated smoother sequences of instabilities ${ }^{19,20}$ and for which theoretical analyses of stability thresholds and time evolution are more tractable.

\section{CONCLUSIONS}

These results demonstrate that particular broadband power spectra and irregular time series coming from an inhomogeneously broadened, single-mode laser are consistent with their representing deterministic motion on a strange attractor of low fractal dimensionality. Further tests of other dimensionalities, entropies, and Lyapunov exponents are required to establish complete certainty of this argument. However, the results clearly demonstrate that these broadband spectra do not arise from stochastic noise sources.

There is a strong temptation to argue that low dimensionalities provide evidence that a small set of collective variables can be used to model the system dynamics. This argument is not directly supported theoretically, however, as the dimensionality gives only the local structure of the attractor and does not provide information on the existence of particular collective varibles or key normal coordinate combinations of the physical variables. Such a reduced set would be a useful simplification from the hundreds of equations now used in modeling time-dependent solutions for inhomogeneously broadened lasers. ${ }^{7}$ Although a small set of key variables is not ruled out by the low-dimensionality results, initial efforts with only a few variables ${ }^{21}$ have produced results inconsistent with those of the more-exact, albeit more cumbersome, models.

\section{ACKNOWLEDGMENTS}

Useful conversations on measures of attractor characteristics with J. D. Farmer, J. P. Gollub, A. S. Wolf, H. L. Swinney, R. Kapral, I. Procaccia, and P. Grassberger greatly aided in interpreting these results. These measurements were made possible by the loan of the Tektronix digitizer by L. M. Narducci and W. Eidson of Drexel University. We also wish to acknowledge the important interfacing and programming assistance and advice of S. P. Adams. The analysis of these large amounts of data was made feasible by a grant of computer time by Widener University.

This research was supported in part by National Science Foundation research grant ECS-82-10263, by a Cottrell Research Grant from Research Corporation, by an Alfred P. Sloan Research Fellowship to N. B. Abraham, and by Helena Rubinstein Foundation Fellowships to J. Abounadi and S. Yong.

* Present address, Department of Physics, University of Rochester, Rochester, New York 14627.

† Department of Physics, Bryn Mawr College, Bryn Mawr, Pennsylvania 19010.

\section{REFERENCES}

1. J. P. Eckmann, Rev. Mod. Phys. 53, 643 (1981).

2. R. S. Gioggia and N. B. Abraham, Phys. Rev. Lett. 51, 650 (1983).

3. H. Haken, Phys. Lett. 53A, 77 (1975).

4. R. Graham, Phys. Lett. 58A, 440 (1976).

5. E. N. Lorenz, J. Atmos. Sci. 21, 130 (1963).

6. L. W. Casperson, IEEE J. Quantum Electron. QE-14, 756 (1978). See also his contribution to Laser Physics, J. D. Harvey and D. F. Walls, eds., Vol. 182 of Lecture Notes in Physics (SpringerVerlag, Berlin, 1983), p. 88.

7. L. W. Casperson, J. Opt. Soc. Am. B 2, 62, 73 (1985); M.-L. Shih, P. W. Milonni and J. R. Ackerhalt, J. Opt. Soc. Am. B 2, 130 (1985); D. K. Bandy, L. M. Narducci, L. A. Lugiato, and N. B. Abraham, J. Opt. Soc. Am. B. 2, 56 (1985).

8. J. D. Farmer, Physica 4D, 566 (1982).

9. P. Grassberger and I. Procaccia, Phys. Rev. A 28, 2591 (1983); Phys. Rev. Lett. 50, 346 (1983); A. Ben Mizrachi, I. Procaccia, and P. Grassberger, Phys. Rev. A 29, 975 (1984).

10. N. B. Abraham, J. P. Gollub, and H. S. Swinney, Physica 11D, 252 (1984).

11. A. S. Wolf and J. Swift, University of Texas, Austin, Texas 78712 (personal communication); J. D. Farmer, in Fluctuations and Sensitivity in Nonequilibrium Systems, W. Horsthemke and D. Kondepudi, eds., Vol. 1 of Proceedings in Physics (SpringerVerlag, Berlin, 1984), p. 172.

12. R. Graham, University of Essen, Essen, Federal Republic of Germany (personal communication).

13. M. Sargent, M. Scully, and W. Lamb, Laser Physics (AddisonWesley, Reading, Mass., 1973); H. Haken, "Laser theory," in Handbuch der Physik (Springer-Verlag, Berlin, 1970), Vol. $\mathrm{XXV} / 2 \mathrm{c}$.

14. H. Whitney, Ann. Math. 37, 645 (1936). 
15. N. H. Packard, J. P. Crutchfield, J. D. Farmer, and R. S. Shaw, Phys. Rev. Lett. 45, 712 (1980).

16. F. Takens, in Proceedings of the Warwick Symposium 1981, D. Rand and L. S. Young, eds. (Springer-Verlag, New York, 1981).

17. A. Brandstater, J. Swift, H. Swinney, A. Wolf, D. Farmer, E. Jen and J. Gutchfield, Phys. Rev. Lett. 51, 1442 (1983); A. Brandstater and H. L. Swinney, in Fluctuations and Sensitivity in Nonequilibrium Systems, W. Horsthemke and D. K. Kondepudi, eds., Vol. 1 of Proceedings in Physics (Springer-Verlag, Berlin, 1984), p. 166; S. Cilberto and J. P. Gollub, J. Fluid Mech. (to be published).

18. R. S. Gioggia and N. B. Abraham in Coherence and Quantum Optics V, L. Mandel and E. Wolf, eds. (Plenum, New York, 1984), p. 563.

19. L. E. Urbach, S.-N. Liu, and N. B. Abraham in Coherence and Quantum Optics V, L. Mandel and E. Wolf, eds. (Plenum, New York, 1984), p. 593.

20. L. M. Hoffer, T. H. Chyba, and N. B. Abraham, J. Opt. Soc. Am. B 2, 102 (1985).

21. R. Graham and Y. Cho, Opt. Commun. 47, 52 (1983).

\section{A. M. Albano}

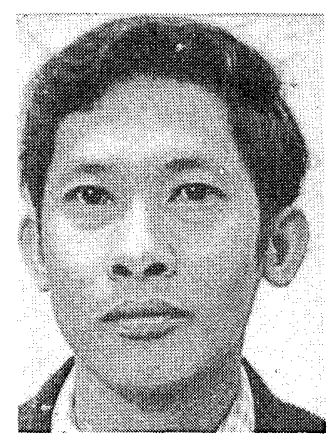

A. M. Albano, who was born in the Philippines on August 2, 1939, is professor of physics at Bryn Mawr College. $\mathrm{He}$ was awarded the B.S. degree in physics by the University of the Philippines in 1959 , the M.S. degree in physics by the University of Iowa in 1964, and the $\mathrm{Ph} . \mathrm{D}$. degree in physics from the State University of New York at Stony Brook in 1969. Although his dissertation topic was high-energy physics, he has, in the past decade, worked on problems in nonequilibrium thermodynamics of interfacial systems, statistical mechanics, molecular dynamics, and nonlinear phenomena. He has taught as an instructor at the University of the Philippines and at SUNY-Stony Brook and has been with Bryn Mawr College since 1970, serving as chairman of the Department of Physics from 1979 to 1984. In 1974-1975 and 1978-1979, he was a visiting physicist at the Lorentz Institute for Theoretical Physics in Leiden, The Netherlands.

\section{J. Abounadi}

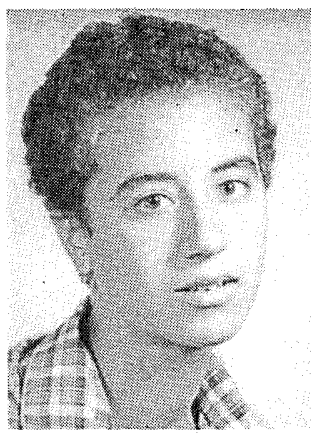

J. Abounadi was born in Marrakesh, Morocco, on July 13, 1966. She is currently a sophomore physics major at Bryn Mawr College and participated in this work under the auspices of a Helena Rubinstein Foundation Summer Research Fellowship (1984).

\section{T. H. Chyba}

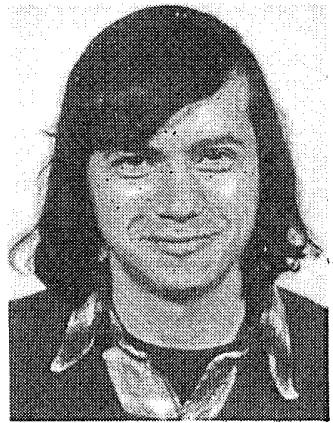

T. H. Chyba was born in Baltimore, Maryland, in September 1956. He received the B.A. degree in mathematics and the B.S. degree in physics from the University of Rochester in 1978 and the M.S. degree in physics from the University of Michigan in 1980. From September 1981 to June 1983, he was an assistant professor of physics at RandolphMacon College in Ashland, Virginia. During the summers of 1982 and 1983, he was a research assistant at Bryn Mawr College. He is now enrolled in the Ph.D. program in the Department of Physics and Astronomy at the University of Rochester. Mr. Chyba is a student member of the Optical Society of America, the American Physiç Society, the American Association of Physics Teachers, and the Society of Physics Students.

\section{E. Searle}

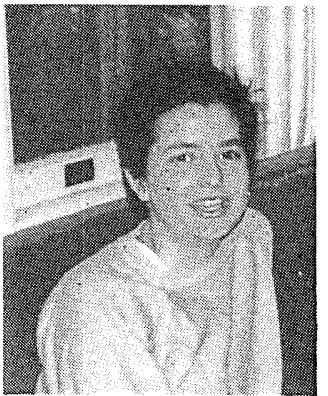

C. E. Searle was born in Berkeley, California on July 19, 1962. She obtained the A.B. degree cum laude, majoring in mathematics and physics with honors in physics, from Bryn Mawr College in 1984. She is currently working at the Centre d'Etudes Nucleaires at Saclay, France, while taking courses for the maitrise in physics and mathematics at the University of Paris-Orsay.

\section{S. Yong}

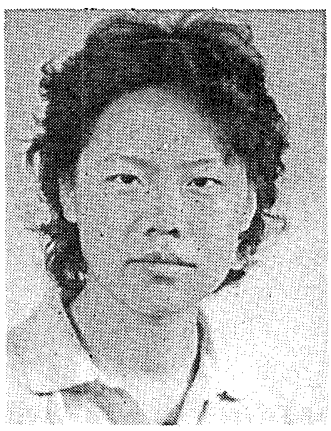

S. Yong was born in Cali, Colombia, on December 31, 1963. She is a junior physics major at Bryn Mawr College and participated in this work as a Helena Rubinstein Foundation Summer Research Fellow in 1984.

\section{R. S. Gioggia}

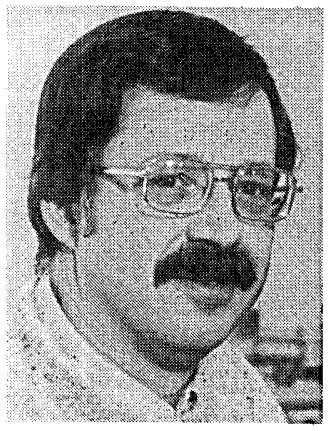

R. S. Gioggia is professor of physics at Widener University. He was born on February 29, 1943, in New York, New York. He obtained the B.S. degree in engineering science from New York University in 1965 and the M.S. degree in physics from Trinity College in Hartford, Connecticut, in 1967. As a graduate student at Bryn Mawr College, where he received the Ph.D. degree in 1975 , he worked on the kinetics of the Curie transition in nickel. For the past several years, his research has dealt mainly with the study of instabilities in laser systems. Dr. Gioggia has taught at Weidener University since 1967 and was chairman of the faculty there from 1970 to 1976 . He is a member of the American Association of Physics Teachers, the American Physical Society, and the Optical Society of America. He was elected a Danforth Foundation Associate in 1980. 